\title{
Meeting of the American Society of Neuroradiology
}

New York, May 9-15, 1987

The 25th anniversary meeting of the American Society of Neuroradiology was held in New York. This world-wide neuroradiologic congress attracts the majority of physicians interested in neuro-imaging techniques, about 1000 of whom meet annually in North America.

Over 200 papers were presented, representing about half of the abstracts submitted. As is usual in neuroThe Aratomy of Speech : Magnetic Resonance Imlaging
(NRI)

AN Gulati, J Astruc, AC Price, JM Dufour, FJ Laine

(hedical college of Virginia, Richmond, VA 23298)

MRI has made it possible to directly image the detai-

led anatomy of the brain in multiple projections. This

has been particularly helpful in the global evalua-

tion of the multiple regions of the brain which are

involved in speech. Speech centers are located adja-

cent to the Sylvian fissure (SF) in the dominant

hemisphere. The classification of speech disorders

is based on pathology in the specific anatomic loca-

tions involved with the verbalization and understan-

ding of speech. The opercular region of the frontal

lobe adjacent to the SF controls the motor function (speaking), whereas the posterior temporal lobe just inferior to the posterior limit of the SF controls the understanding of speech (interpretation) and $v i s u a l$ speech(reading). These areas are all interconnected by white matter tracts in the dominant hemisphere, as well as commisural fibers relaying information to the dominant hemisphere. We will describe and show MRI images in multiple projections together with correlative gros anatomic sections. imaging, and in keeping with neuroradiologic traditions, many of the papers presented were of high anatomic quality. By permission of $\mathrm{J}$ Taveras and of the publishers, Williams and Wilkins, we present some of the papers dealing with anatomic topics presented during this meeting.

P Lasjaunias

\begin{abstract}
$M \mathbb{R}$ of pacchionian Granulations
BD Pressman, MJ Lotysch (Cedars Sinai Médical Center, Los Angeles, CA 90048)

Pacchionian granulations are extensions of arachnoidal tissue into major venous sinuses, dural lakes and veins Characteristic depressions or erosions of the inner table occur relatively frequently. Much less commonly these may produce florid erosions of the inner table and diploe, and may thin, bulge on even erode through the outer table. They may mimic neoplastic or other significant pathology on skull radiographs or CT. We performed CT on a 77 year old woman evaluated for acute disorientation. There were multiple bilateral paramedial erosions of the inner and middle table with marked thinning of the outer table of the occipital bone. MR showed that this was secondary to intra osseous extension of a grape-like cluster of structures contiguous with the peripheral surface of the brain and having $M R$ signal equivalent to $C S F$ on all sequences. The $M R$ clearly demonstrated that this was not a primary osseous lesion, and that there was no associated intra-axial pathological process and no mass effect on the brain. Although there is no pathological proof, this appearance of lobulated CSF containing structures arising from the peripheral margin of the brain in an appropriate location may fairly be consideral typical of the MR appearance of Pacchionian granulations.
\end{abstract}

\section{Erratum}

The 5th Asian and Pacifie Congress of Radiology was erroneously assigned to Sendai in Number 2 of this volume. This congress was actually held in Seoul in Korea between 21 and 25 September 1987. We ask the organizers of this congress to accept our apologies for this unintentional typographic error. 